\title{
La gestión de conflictos y competencia territorial vistas desde la gestión pública
}

\section{Conflict management and territorial competition seen from public management}

\author{
Marianella Pinella Odar ${ }^{1}$ \\ Autor Independiente \\ marianellapinella@yahoo.es \\ https://orcid.org/0000-0002-9983-9476
}

Aceptado: 03/12/2019

\section{RESUMEN}

Al estudiar el tema de gobernabilidad distrital y límites podemos pensar en los problemas y controversias que el tema genera, como: el debilitamiento de recaudación en impuesto predial, que son ingresos monetarios administrados y fiscalizados por las alcaldías, muy aparte de los perjuicios sociales a nivel ciudadano. El conflicto limítrofe existente entre los distritos de Magdalena del Mar y San Isidro, el cual lleva ya varios años sin resolver, y afecta el desarrollo social y económico, alterando el ambiente social de armonía y la ejecución de obras públicas y servicios municipales que se ponen en riesgo; como la seguridad ciudadana, entre otros. Los intentos de solución han sido exiguos para resolver el conflicto de parte del Estado, solo logrando dividir aún más a los habitantes de ambos distritos. Nuestro estudio revela los puntos débiles que existen en la presentación de alternativas para solucionar el problema, primero: la ausencia de una herramienta de gestión de políticas públicas y de respeto al estado de derecho, en donde las autoridades al mando de ambos distritos se sometan a un ente decisorio técnico como el Instituto Geofísico del Perú, organismo que mediante coordenadas geográficas puede establecer definitivamente los límites de los territorios en conflicto.

(C) Los autores. Este artículo es publicado por Pensamiento Crítico de la Facultad de Ciencias Económicas, Universidad Nacional Mayor de San Marcos. Este es un artículo de acceso abierto, distribuido bajo los términos de la licencia Creative Commons Atribucion - No Comercia_Compartir Igual 4.0 Internacional. (http:// creativecommons.org/licenses/by-nc-sa/4.0/) que permite el uso no comercial, distribución y reproducción en cualquier medio, siempre que la obra original sea debidamente citada. 
Palabras claves: Gestión de conflicto; diferenciales interjurisdiccionales; economía pública.

Códigos (JEL): Gestión de conflicto (D74), diferenciales interjurisdiccionales (H73), economía pública (H80).

\section{ABSTRACT}

Understanding that the problematic of the district limits generates the weakening of collection in property tax, main municipal tax administered by the municipalities according to the Organic Law of municipalities. This is the specific case of the border conflict between the districts of Magdalena del Mar and San Isidro, a situation that has long been the case, and affects the social and economic aspect of the populations and residents, limiting the execution of public works and municipal services such as citizen security in the affected districts. Attempts to solve the conflict by the State only polarize the citizens of both communes and stop living in harmony as if they were bordering countries. The study auscultates the negative axes and how the lack of a public policy management tool increasingly sharpens a political situation and respect for the rule of law, where the authorities involved must submit to a technical decision-making body as is the specific case of the National Geographical Institute, which, by means of geographical coordinates, can delimit the territories in conflict. Keywords: Conflict management; interjurisdictional differentials; public economy. Codice (JEL): Conflict management (D74), interjurisdictional differentials (H73), public economy (H80). 


\section{Introducción}

Podemos ver en la realidad actual de nuestro país el poco interés que existe de solucionar conflictos entre poblaciones, ya sea en provincias o distritos del Perú, y que en casi el75\% de ellos existan problemas limítrofes. Por tal razón en el presente estudio se aborda presentando posibles soluciones que se ocupen del problema y termine con esta antigua controversia entre dos de los distritos más representativos de la ciudad de Lima como lo son Magdalena del Mar y San Isidro, cuyas demarcaciones no habrían sido precisadas correctamente.

Algunos de los problemas que ha traído la falta de delimitación clara del territorio han sido causados por la falta de actualizaciones a leyes preexistentes y el desconocimiento masivo de la población acerca de sus límites territoriales ha generado inconvenientes graves como el enfrentamiento con palos y piedras que se dio entre las autoridades municipales encargadas justamente de preservar el orden y la seguridad de los distritos. Entre otras razones, el incipiente análisis técnico de este suceso ocurrió porque existe una falta de acuerdos entre las autoridades competentes, que permita a la población comprender los conflictos territoriales.

El presente estudio tendrá como marco de estudio el análisis de algunos elementos que impulsan el conflicto, determinando cómo se ha llevado a cabo la "negociación" de conflictos de competencia territorial entre Magdalena y San Isidro a lo largo de su historia, definiendo el territorio municipal, la tipificación de las divisiones político territoriales (geométrico, el natural y el mixto); también se estudiará el papel que ha cumplido la Oficina Nacional de Dialogo y Sostenibilidad y el marco técnico normativo; como también se analizará la historia del conflicto, y vemos como su entrampamiento, obedece a una lógica de politización que promueve la dilación de una solución equitativa en los diferentes niveles, administrativo y económico, del conflicto.

De otro lado se muestra la metodología de la investigación utilizada, presentando la hipótesis, objetivos y los resultados, que a su vez nos conducen a los argumentos usados y a los elementos que representan al conflicto; y finalmente concluimos: que el ente competente que debería ejecutar una delimitación equitativa es el Instituto Geofísico del Perú, a través del establecimiento de coordenadas geográficas. 


\section{Metodología}

Esta investigación es de tipo descriptiva, porque analiza y describe las variables de estudio presentadas. La investigación se perfila al conocimiento de la realidad tal y como se presenta, en una situación espacio temporal dado, es decir, requiere detallar las características resaltantes de los grupos de estudio o cualquier otro fenómeno que sea sometido a estudio.

El diseño de la investigación presentada es no experimental y transversal. Se entiende como no experimental porque no se manejaron hipótesis improvisadas. Es transversal, porque se mide solo una vez las variables de estudio.

La población que fue estudiada, estuvo conformada por los vecinos de los distritos de Magdalena del Mar y San Isidro, la muestra la constituyeron 120 personas que forman partes de ambos distritos, la muestra fue de tipo "no probabilístico". A los cuales se les aplicó una encuesta determinando el nivel de confiabilidad Alfa de Cronbach, valor alcanzado 956 para gestión de conflictos de competencia territorial y 974 gestión pública, ambos resultados indican una alta confiabilidad. Para el análisis estadístico se empleó el software estadístico SPSS.V21.

\section{Hipótesis general}

Ausencia de una visión de Gestión Municipal que permita entender los conflictos de competencia territorial en la perspectiva de la Gestión Pública.

\section{Hipótesis específicas}

El Estado peruano ha establecido criterios asistemáticos para generar tipologías de conflictos limítrofes que se presentan en el ordenamiento del territorio, en la concesión de recursos y la administración fiscal.

El conflicto limítrofe entre la Municipalidad de Magdalena del Mar y la de San Isidro incide en el aspecto económico. 


\section{Marco Teórico}

\section{Gestión de Conflictos de Competencia Territorial}

\section{Territorio municipal}

Un territorio municipal, es un nombre vinculado a un municipio, el cual se refiere a una entidad territorial que forma parte de una división administrativa de un país, asimismo se conoce como municipalidad al gobierno municipal. Podemos ampliar nuestro conocimiento del tema refiriéndonos a los siguientes autores:

"La organización territorial del Estado se encuentra contenida en el artículo $137^{\circ}$ de nuestra Carta Magna, que determina que el Estado se organiza territorialmente en Municipios, Provincias y en Comunidades Autónomas". (Sánchez, 2003, p. 78).

De otro lado, el territorio municipal, según Aldo Sehwerert Ferrer, citado por García (2009, p.12), que “...sin territorio hay municipio; de allí que se diga que el municipio no está limitado, sino que está constituido por el territorio, que le pertenece jurisdiccionalmente".

El Poder Municipal es la autoridad de mando que tiene a cargo un Municipio. Esta persona elegida democráticamente suele tener a su cargo la responsabilidad de administrar un presupuesto público, el cual es provisto por el Gobierno Central del país para hacer frente a gastos e inversiones en obras públicas como por ejemplo la limpieza de la ciudad o promocionar campañas turísticas, entre otras actividades.

La Constitución de nuestro país garantiza autonomía para las municipalidades y personalidad jurídica para la realización de sus respectivas obras, ya que cuenta con instituciones y organismos que le entregan el poder de ejecutar medidas de gobierno dentro del municipio en cuestión, y le dan la posibilidad de elegir sus propias autoridades, gestionar cualquier materia que sea de su competencia y generar ingresos propios que puedan administrar con libertad, e invertirlos en los proyectos que considere adecuados dentro de su jurisdicción. 


\section{Controversia y problemas de la delimitación territorial}

Los temas como delimitación territorial, política y administrativa son instrumentos necesarios para la ordenación de los estados como de sus jurisdicciones. La división de los Estados en sus áreas políticas y administrativas con sus límites, se corresponden a sus formas topográficas, así como a las relaciones productivas, económicas, sociales y gubernamentales que se dan en su ámbito.

Según nos señala el autor Yensen (2017), los problemas asociados a delimitación territorial, están basados en disputas entre un territorio que reclama tierra entre su frontera y el del territorio vecino, debido a alguna cualidad que la tierra posee.

Hemos podido observar en el Perú, a lo largo de estos años que se han suscitado una serie de conflictos asociados a problemas de demarcación distrital, el Estado centralista ha generado espacios con características dispares, unos más beneficiados como Lima y zonas de la costa, y otros espacios menos beneficiados, como los Andes y la Amazonía, donde es menor la presencia del Estado. Según el INEI (2017), para el Censo Nacional de 2016 se concluyó que de los 1874 distritos que conforman el territorio peruano, 1460 de ellos tienen problemas limítrofes. Tras una revisión de las delimitaciones político-administrativas actuales, se verificó la existencia de graves problemas e incoherencias en diferentes contextos de acuerdo con la Dirección Nacional de Demarcación Territorial de la Presidencia de Consejo de Ministros (PCM) y por el Instituto Metropolitano de Planificación (IMP).

¿Cuál es la razón por la que más de un 75\% de los distritos peruanos tengan conflictos entre sus limítrofes?, pues creemos que se debe principalmente a que la mayoría de estos lugares fueron creados antes de que existiera una normativa que delimitara claramente un límite entre ellos, ya que la creación de la mayoría de los distritos se originó en la época colonial, con base en accidentes geográficos, decisiones políticas arbitrarias, identidad sociocultural de la población o por características fisiográficas del paisaje como ríos, colinas, crestas de montañas, tipologías que eran fáciles de identificar desde ambos lados de la frontera distrital y que en la actualidad ya no se encuentran presentes. 
Los entes municipales son los encargados de resolver dicha problemática y justamente son ellos los que muestran un desconocimiento insólito acerca de los hechos históricos que condujeron a la distribución territorial, además de mostrarse en una situación de ignorar y evadir la responsabilidad de corrección, porque no son los suficientemente graves para motivar las autoridades a buscar una solución, ya que es más lo que pierden que lo que pueden llegar a ganar.

\section{Nuevo enfoque de sistematización de sectores urbanos}

La visión de desarrollo y la implementación de grandes proyectos urbanos, pasa por un proceso de planificación urbana que tiene por objeto estimular las transformaciones de una parte o la totalidad de la ciudad, incluye su forma física, pero también su imagen para posicionarla favorablemente en la competencia interurbana (dimensión proyecto-objeto).

Pero aparte de estos objetivos comunes, estas operaciones también están implementando un modo de acción común: lo que se llamará "enfoque de proyecto" (dimensión proyecto-proceso). (Boutinet, 1993, p.27)

En primer lugar, se debe tener en cuenta, a través del proceso de proyecto, los actores de las políticas urbanas ya no aprehenden a la ciudad como un continuo espacio neutral a desarrollar, pero como un lugar específico para valorar y una comunidad para movilizarse en el marco de una competencia territorial cada vez más exigente. Entonces, a través del proyecto, es una racionalidad de acción que es decididamente interaccionista, inductiva y incrementalista que es privilegiado. Los actores de la política urbana ahora saben que ellos actúan en un contexto incierto marcado notablemente por el cambio de tamaño del rol redistribuidor del estado, la escasez de recursos públicos, la mayor exposición de las ciudades, las exigencias de la situación económica y la creciente competencia territorial.

En los enfoques del proyecto, los actores no abandonan la preparación del futuro de la ciudad urbana pero saben que los procesos de transformación urbana operan en mucho tiempo y que este tiempo necesariamente implica un cambio en el stock de recursos disponibles, por lo tanto, deben decidir cómo van asumir los nuevos retos. (Boutinet, 1993, p.29) 
Las políticas urbanas son únicas en el sentido de que no forman parte de acción pública en la cual las divisiones ideológicas se cristalizan muy claramente, lo particularmente difícil de identificar políticas y políticas urbanas de izquierda y de derecha en el aspecto urbano. Las políticas urbanas generalmente tienen una dimensión técnica.

Además, rara vez el tema de la controversia política a nivel nacional genera líneas divisorias muy claras, incluso dentro de los dos "campos", las prácticas de los funcionarios electos en la planificación urbana son muy heterogéneos de una ciudad a otra. Finalmente, es una apuesta segura que los propios votantes urbanos no operen su elección electoral de acuerdo con un mapa mental que distinga muy claramente, las propuestas de planificación de la izquierda de las de la derecha.

Los enfoques del proyecto no hacen nada para disipar esta vaguedad ideológica que rodea las Políticas urbanas. Por el contrario, tienden a mantenerlo. El proyecto tiene, por un lado, encantos de un modo de acción antitecnócrata, culturalista y localista que puede complacer izquierda, especialmente en su versión "autogestionada". (Boutinet, 1993)

Cuando se cumplen las condiciones políticas e institucionales adecuadas, y con liderazgo y fuerte compromiso, planeando el desarrollo puede acelerar el crecimiento económico y la transformación.

Las políticas pueden guiar la concentración de inversiones públicas y sector privado en sectores estratégicos para lograr un desarrollo significativo. Al mismo tiempo, se necesita apoyo político para garantizar una mejora constante infraestructura y el entorno comercial e institucional para apoyar innovación tecnológica, modernización industrial y diversificación.

\section{El problema del conflicto limítrofe entre Magdalena del Mar y San Isidro.}

“Art. 3o.- El distrito de Magdalena del Mar tendrá por capital el pueblo de su nombre y estará limitado por el Océano Pacífico, una línea recta perpendicular a la playa, pasando por la casa del fundo Santa Cruz, y otra que partiendo de ese punto encuentre la intersección del camino al Callao, con el lindero entre San Miguel y la Magdalena del Mar”. 
El distrito limítrofe de San Isidro se creó el 24 de abril de 1931 (once años después que su vecino), mediante un decreto ley del distrito de Miraflores, del cual se separa. Principalmente formaba parte del noroeste son con las dos Magdalenas existentes (Magdalena del Mar y Magdalena Vieja o Pueblo Libre). Los límites del nuevo distrito de San Isidro se conforman por la línea que parte desde el ovalo Bielich (hoy Óvalo Gutiérrez) al cuartel San Martin. Ciertamente esta línea que se dirige al cuartel San Martin tiene una valla legalmente infranqueable y es la recta que va desde la Casa Hacienda Santa Cruz hasta el Océano Pacífico como lo determina la ley de creación de Magdalena del Mar, la misma que no ha sufrido hasta la fecha modificación alguna ni ha sido derogada ni anulada salvo en el tramo específicamente determinado por el decreto ley no.11170 del 19 de setiembre de 1949 que instaura límites del distrito de Miraflores.

En el distrito de San Isidro, las propiedades se hallan valorizadas a mayor precio que en Magdalena del Mar; sin embargo, el vecino puede tributar o pedir licencias donde más le beneficie. Si va al distrito de San Isidro, allí le autorizarán el desarrollo de una construcción de quince pisos, lo que en Magdalena no sería posible por tratarse de una zona residencial. A consecuencia de esto, la demarcación territorial se ha politizado generando controversia en lugar de armonía entre los vecinos.

Por algunas de estas señales se ha visto que el distrito de San Isidro tiene la intención de expandirse y apropiarse de terrenos que no le corresponden por determinación de la ley, usando argumentos carentes de legalidad y muy buenas relaciones públicas; además de amenazar a los vecinos (Magdalena del Mar) con amenazas tributarias.

Debemos señalar que es ilegal afirmar que, por decretos o resoluciones ministeriales, por decretos de alcaldía o inscripciones regístrales se pueda modificar una ley expresa que fija límites determinados. - Conforme el Código Civil: "Ninguna Ley se deroga sino por otra Ley"; ya que al no haber sido derogada la Ley No. 4101 que creo el Distrito de Magdalena del Mar y que fijó sus límites, están vigentes en todo extremo dichos límites y derechos.

El Art. 51ํ de la Constitución Política de la República (1993) señala textualmente: 
"La constitución prevalece sobre toda norma legal; la ley sobre las normas de menor jerarquía y así sucesivamente. - la publicidad es esencial para toda norma del estado".

Para realizar una delimitación justa se debe recurrir al Instituto Geofísico del Perú, quien, a través especialistas en historia y geografía, podrán establecer la fijación de coordenadas geográficas, hallando el punto de inflexión, donde se registran la latitud y longitud exacta y se coloca un hito en ese lugar, para de esta manera poder resolver la problemática instaurada; ya que la ignorancia de las autoridades municipales es evidente.

\section{Aspecto económico}

Acudiendo a la consulta del INEI (2018), podemos encontrar información referida al crecimiento económico sostenido durante un largo periodo, se ve respaldado por una coherencia en las políticas macroeconómicas emprendidas por los últimos gobiernos, de modo tal que el Perú, registra uno de los índices más bajos en relación con la región con las tasas bajas, con un PBI de $2.2 \%$, con un incremento significativo en la demanda interna que alcanza el 5\%, lo que se ve fortalecido por el aspecto cambiario, con una moneda fuerte en relación con el dólar, teniendo una calificación de riesgo país, baja pese a los acontecimiento políticos de los últimos trimestres, las exportaciones han contribuido a mantener los niveles adecuados de recaudación, fortaleciendo la imagen del país a nivel internacional.

El distrito de Magdalena del Mar, ha sido favorecido por la estabilidad macroeconómica y por el crecimiento que ha tenido en los últimos años el país, pues en la actualidad tiene un importante número de edificios en construcción, favorecido en parte por el "boom inmobiliario" que ha determinado la venta de inmuebles antiguos, debido a que magdalena es aún un distrito con un buen porcentaje de viviendas antiguas y familiares. Si comparamos con el distrito de San Isidro, su situación es contraria, tiene un gran porcentaje de edificios; a parte el crecimiento ha estado en decadencia lo que indica que últimamente ha estado estancada su economía, no ha progresado como en otros años.

Los dos distritos están creciendo económicamente, de tal modo que existen controversias con las fronteras distritales que estos poseen. Cada distrito quiere crecer aún más para tener una mejor economía y debido 
a eso han surgido diversos problemas como comprar en Magdalena a un precio por debajo y registrarlo en San Isidro de manera que el terreno adquiera más valor, esto se aplica especialmente a los comerciantes, ya que San Isidro por ser una zona comercial se pagan unos impuestos elevados comparados a Magdalena del Mar, es por ello que parte de la población que no quiere pertenecer a San Isidro.

Las municipalidades son entes estatales que manejan sus recursos propios generados principalmente por la recaudación del impuesto predial que brindan los vecinos. La localización de los predios, el cobro y la recaudación de impuestos son los elementos que intervienen en gran medida en los conflictos limítrofes municipales.

\section{Aspecto administrativo}

Según el autor Quevedo (1998), las Gerencias Municipales son los órganos más altos, en la estructura orgánica, siendo estos especializados en el aspecto técnico administrativo, en diversas áreas; rentas, fiscalización, administración tributaria que conciernen a la jurisdicción. Estas gerencias trabajan dando cuenta al planeamiento anual de la municipalidad, así como las políticas propias de la Autoridad municipal, el Alcalde.

Las estrategias operativas y preventivas realizadas por las instituciones representativas y las comunidades organizadas, por el sector empresarial y los comités distritales de seguridad ciudadana, en el marco de la participación ciudadana. De otra parte, diversas subgerencias, como Catastro municipal, Obras públicas, Licencias, Relaciones públicas, Registro civil, Cultura y deportes.

La población de cada distrito debe gozar de dichos beneficios, no obstante, cada distrito tiene ciertas particularidades al momento de llevar a cabo estrategias para alcanzar los objetivos distritales; pero la disputa entre las limítrofes hace que eso sea imposible ya que existe un conflicto de intereses entre estos, en los que influyen las zonas industriales, comerciales y pobladores que viven dentro de la problemática y sus impuestos.

Para la entrega de mayores facilidades en otorgamiento de licencias, el distrito de San Isidro ha realizado un tipo de zonificación, que no es permitida en Magdalena, por tratarse de una zona residencial, permitiendo 
el desarrollo de diferentes empresas y comercios, esto situación hace que exista una cierta población que quiera pertenecer a San Isidro, y otra que simplemente desea pertenecer a Magdalena.

\section{Resultados y Discusión}

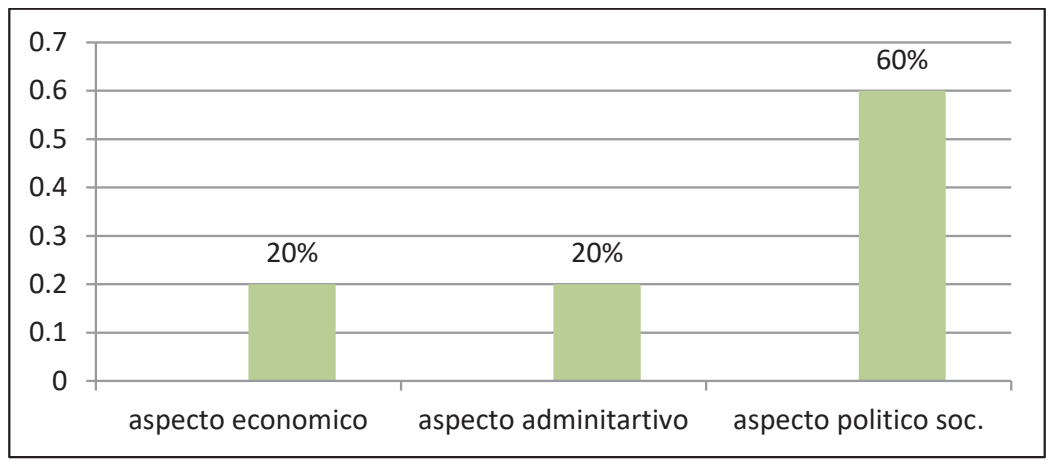

Figura 1. Gestión de conflictos de competencia territorial San Isidro Fuente. Resultados del instrumento, Gestión de conflictos de competencia territorial.

Observando los resultados del cuadro realizado, podemos afirmar que el $60 \%$ de los ciudadanos encuestados piensa que se trata de un problema de índole político-social, mientras que el 20\% piensa que es un tema económico, y el restante $20 \%$ considera que se trata de un problema de índole administrativa.

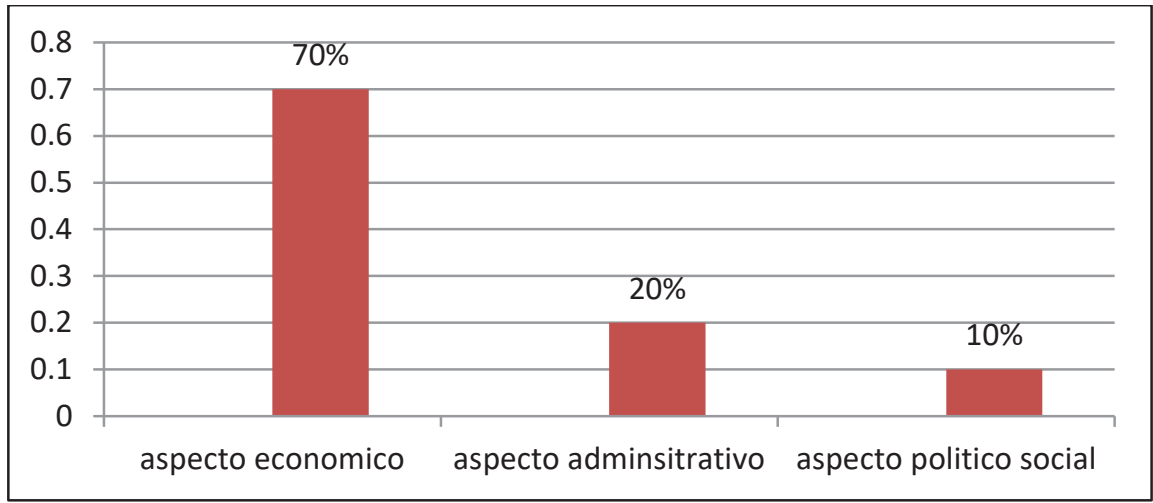

Figura 2. Distrito de Magdalena del Mar.

Fuente. Resultados del instrumento, Gestión de conflictos de competencia territorial. 
Los ciudadanos entrevistados en el distrito de Magdalena del Mar piensan que el problema es de índole económica; un 20\% considera que es un tema de aspecto administrativo, y el 10\% lo considera desde una perspectiva político-social.

Análisis: En los gráficos elaborados se muestra que los ciudadanos de cada municipio tienen diferente percepción con respecto al tema de límites territoriales con su vecino distrito; de una parte, Magdalena del Mar considera que se trata de un tema de índole económico, mientras que los pobladores de San Isidro consideran que es un tema de índole político-social. La percepción de los ciudadanos de Magdalena del Mar es congruente con lo alcanzado por su gestión municipal con respecto a los inmuebles que se encuentran en la zona de conflicto. Pese a este problema, este municipio recibe grandes sumas de dinero por impuestos y licencias.

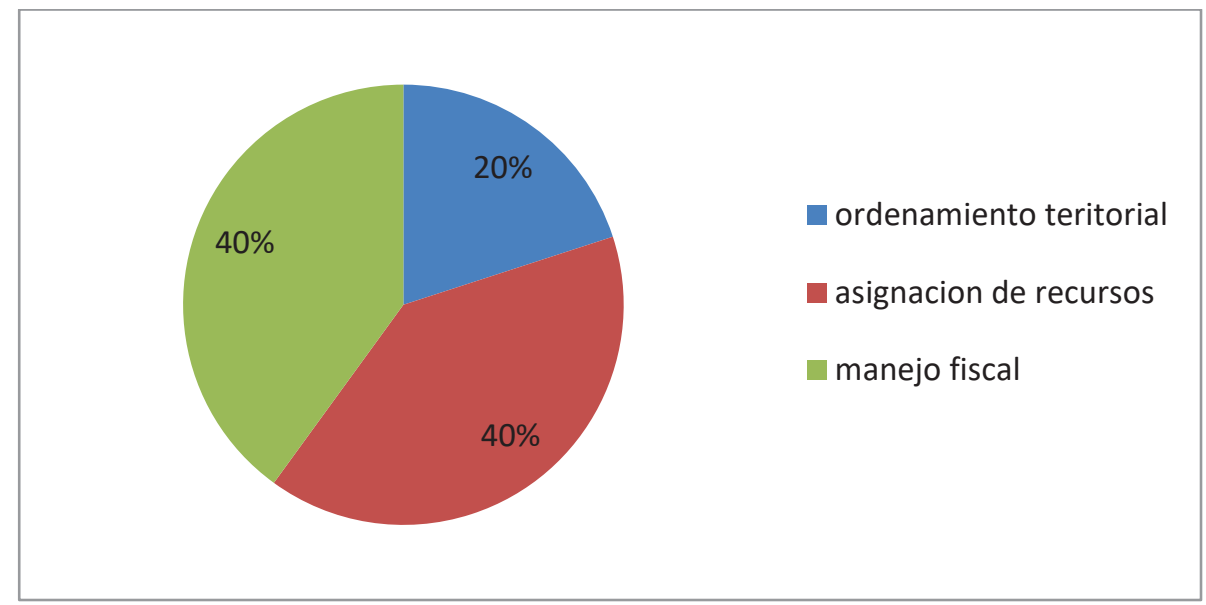

Figura 3. Percepción de las autoridades del distrito de San Isidro.

Fuente. Resultados del instrumento, Gestión de conflictos de competencia territorial.

Del total de ciudadanos encuestados en el distrito de San Isidro, un $20 \%$ cree que las autoridades del municipio han dirigido adecuadamente el tema del ordenamiento territorial; mientras que solo un $40 \%$ considera que existe transparencia en la asignación de recursos, y el $40 \%$ cree que el manejo fiscal es el adecuado. 


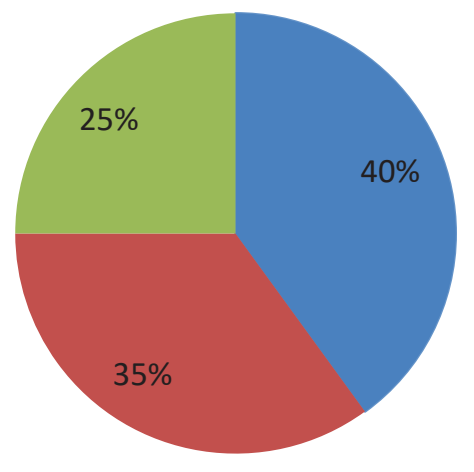

ordenamiento territorial $40 \%$

Asignacion de recursos $35 \%$

Manejo fiscal 25\%

Figura 4. Percepción de las autoridades del distrito de Magdalena del Mar. Fuente. Resultados del instrumento, Gestión de conflictos de competencia territorial.

Según los ciudadanos encuestados en el distrito de Madalena del Mar, un $40 \%$ cree que las autoridades del municipio han manejado bien el tema del ordenamiento territorial; mientras que un 35\% cree que existe poca claridad en la asignación de recursos, y el 25\% piensa que el manejo fiscal es inadecuado.

Análisis: De los gráficos anteriormente vistos, se concluye que el Municipio de Magdalena del Mar ha salido favorecido del problema limítrofe con su vecino de San Isidro, en tanto que la mayor cantidad de propietarios de la zona ha inscrito su propiedad en Madalena del Mar, realizando los pagos concernientes al impuesto predial, contribuciones y serenazgo en este distrito.

\section{Discusión}

Los autores Ramírez, Salgado y Huapaya (2012), identifican los factores que limitan la gestión de autoridades y funcionarios en el caso específico de la Municipalidad Provincial de Abancay, para generar políticas efectivas de impulso del Desarrollo Económico Local (DEL), en el período 20072009, teniendo en cuenta que el tema se ubica a nivel de las actitudes y comportamientos de los actores involucrados y que estos se insertan en 
los intereses, individuales o de grupos diversos, que condicionan el acercamiento o compromiso con el gobierno municipal. La investigación se enfocó en las percepciones de estos.

Yensen (2017), nos entrega un análisis cierto de la situación, que en parte gira en torno a una ineficiencia en la toma de decisiones provocada por el desconocimiento técnico e histórico acerca del problema de demarcación territorial, es decir, las autoridades actuales y anteriores no han estado lo suficientemente capacitadas ni informadas acerca de los orígenes del conflicto. Este desconocimiento se hace visible en el ámbito jurídico, manifestándose carencias en el ámbito normativo, a partir de las cuales se pueden proponer soluciones entre las partes. La problemática puede ser analizada bajo la perspectiva de las actitudes, comportamientos y capacidades de las autoridades locales, como variables dentro del análisis de conflictos por disputas territoriales.

Los problemas de evasión fiscal, los cuales se tornan más complejos porque las confusiones limítrofes pueden jugar a esconder a los evasores de impuestos entre los vecinos confundidos, quienes creen, a veces, que el municipio de su elección atiende de preferencia a los que pagan sus impuestos que a los que mantienen una disputa activa sobre la zona. (Yensen, 2017, p. 143).

Al repasar estos antecedentes, nos damos cuenta que es Yensen (2017) la fuente que más consultada acerca de los aspectos diacrónicos de la problemática de límites territoriales en los distritos del cono norte. Observamos cómo el origen del problema es la falta de criterios en la autoridad que originalmente detenta la demarcación, lo cual no solo obedece a desinformación, sino a comportamientos y conflictos de intereses, quienes de algún modo se ven beneficiados con la carencia de una jurisdicción definida territorial y legalmente. Para la determinación de estos límites distritales, el ente competente es el Instituto Geofísico del Perú, de cuya opinión autorizada dependerá la decisión final que asuman las autoridades municipales.

\section{Conclusiones y Apreciaciones Finales}

Estos conflictos desatados entre ambas municipalidades son de larga data. Ha sido el llamado "boom de la construcción" de los distritos de 
Magdalena del Mar y San Isidro, la causa principal de las pugnas por acceder a las rentas que fluyen al Estado, especialmente del impuesto predial, licencias de construcción y funcionamiento que se distribuyen en los territorios en donde se ubican. Por esta razón en el transcurso de los últimos años, se han identificado más de 500 controversias que enfrentan a regiones, provincias o distritos, que luchan por demarcar territorios de manera que se les permita obtener una porción de los ingresos.

En la actualidad la Gestión Municipal no tiene una visión clara acerca de la competencia territorial desde una perspectiva de gestión pública. La gestión municipal, por lo observado debilita la recaudación tributaria y ve mermados sus ingresos por impuesto predial, ante una indefinición consiguiente en el padrón o catastro y la administración de los servicios y las rentas públicas.

Es vital apartar la politización por parte del Estado para generar la solución demarcatoria definitiva, ya que la mala gestión de ambas municipalidades

Por esta razón se recomienda que el ente competente para un arbitraje correcto sea el Instituto Geofísico, que deberá instaurar las coordenadas geográficas reales y justas de manera equidistante sobre el área en conflicto, dividiéndola en partes lo más iguales posible para cada distrito. Esto, gracias a la tecnología del GPS y a métodos geodésicos y topográficos. Concluyendo con la elaboración de una carta digital de los planos distritales.

Asimismo, será necesario un trabajo de información en todos los niveles y la implementación de mecanismos de participación que puedan generar la solución del conflicto, buscando el modo de consulta a las bases vecinales, promoviendo el diálogo amplio vecinal e inter-distrital, levantando inquietudes e identificando necesidades y quejas, consolidando los criterios que se deberán tener en cuenta ante la perentoria demarcación mediante dirimentes técnicos, como lo es el IGP (Instituto Geofísico del Perú).

\section{Referencias Bibliográficas}

Boutinet J. (1993), Antropología del proyecto, París. Edit. Euxio

Congreso de la República Peruana (1920). Ley № 4101. División del Distrito de Magdalena, en la Provincia de Lima.p.12 
Congreso de la República (1949). Ley № 11170. Decreto Ley que señala los límites de los distritos de Miraflores y Surquillo de la Provincia de Lima. p.32

Constitución Política del Perú de 1993 - Editora Perú. p.172.

INEI (2017). Censo Nacional 2016: XII de Población, VII de Vivienda y III de Comunidades Indígenas. Infraestructura estadística. p.226

INEI (2018). Una mirada a Lima Metropolitana. Lima- Perú: Instituto Nacional de Estadísticas.p.231

García, A. (2009). El derecho municipal en México. El municipio, base fundamental del federalismo en México. México- Porrúa. p.14.

Quevedo, Wilberto (1998). "Enfoque Gerencial de la Municipalidad", Editorial San Marcos. Lima - Perú. Pp: 10 - 98.

Ramírez, Santos; Salgado, Carlos y Huapaya, Jorge. (2012) Tesis "Factores que limitan la gestión de las políticas de desarrollo económico en la municipalidad de Abancay en el periodo 2007-2009", para optar el grado de Magister en Gerencia Social. Pontificia Universidad Católica del Perú. p. 102.

Sánchez, A. (2003). Principios de admiración pública. Madrid- España: spain.p.74

Yensen, Erica (2017) Tesis Demarcación y conflictos territoriales; análisis del conflicto limítrofe entre los distritos de San Martín de Porres, Independencia y Comas. Pontificia Universidad católica del Perú. P. 186.

\section{Notas al final}

1 El título del artículo se origina de la tesis vinculado planteamiento del problema y actores involucrados.

2 Especialista en Planificación e Indicadores ODS, marianellapinella@yahoo.es, 945059977 
